\title{
Performance of improved energy detector based cooperative spectrum sensing over Hoyt and Rician faded channels
}

\author{
Srinivas Nallagonda, Aniruddha Chandra ${ }^{\mathrm{a})}$, \\ Sanjay Dhar Roy, and Sumit Kundu
}

Electronics and Communication Engineering Department, National Institute of Technology, Durgapur, India

a) aniruddha.chandra@ieee.org

\begin{abstract}
In this letter, we develop closed-form analytical expressions for the false alarm and missed detection probabilities of a cooperative spectrum sensing (CSS) scheme using cognitive radio (CR) nodes that are equipped with improved energy detectors (IEDs), employ receive antenna diversity, and operate over Hoyt (Nakagami- $q$ ) or Rician (Nakagami- $n$ ) faded sensing channels. The derived expressions are validated through extensive simulations.
\end{abstract}

Keywords: cooperative spectrum sensing, improved energy detector, multiple antennas, fading channels, total error rate

Classification: Wireless Communication Technologies

\section{References}

[1] A. Singh, M. R. Bhatnagar, and R. K. Mallik, "Cooperative spectrum sensing in multiple antenna based cognitive radio network using an improved energy detector," IEEE Commun. Lett., vol. 16, no. 1, pp. 64-67, Jan. 2012.

[2] F. F. Digham, M.-S. Alouini, and M. K. Simon, "On the energy detection of unknown signals over fading channels," Proc. IEEE ICC, Anchorage, USA, vol. 5, pp. 3575-3579, May 2003.

[3] A. H. Nuttall, "Some integrals involving the $Q_{M}$ function," IEEE Trans. Inf. Theory, vol. 21, no. 1, pp. 95-96, Jan. 1975.

[4] H. L. Van Trees, Detection, Estimation, and Modulation Theory, Part I, Wiley, 2013.

[5] A. Chandra, C. Bose, and M. K. Bose, "Symbol error probability of noncoherent $M$-ary frequency shift keying with postdetection selection and switched combining over Hoyt fading channel," IET Commun., vol. 6, no. 12, pp. 1692-1701, Aug. 2012.

[6] M. K. Simon and M.-S. Alouini, Digital Communication over Fading Channels, Wiley, 2nd ed., 2005. 


\section{Introduction}

Cooperative spectrum sensing (CSS) helps to achieve better management of scarce and under-utilized radio spectrum in a cognitive radio (CR) network. The performance of CSS is further improved by using an improved energy detector (IED) at CR terminals [1] in contrast to conventional energy detector [2]. Due to the analytical tractability, existing literature mostly considers the sensing channel as Rayleigh faded while evaluating the detection performance. Hoyt (or Nakagami- $q$ ) and Rician (or Nakagami- $n$ ) statistics allow us to characterize more-severe-than-Rayleigh (Hoyt) and less-severethan-Rayleigh (Rician) fading scenarios. Moreover, these two models can be used to represent the good and the bad states of a two state Gilbert Elliot model. Motivated by this fact, we analytically investigate spectrum sensing performance of a CSS scheme involving IED based CR nodes with multiple antennas, where the sensing is performed over either a Hoyt or a Rician fading environment.

\section{Analytical framework}

Let us consider a CSS scheme where $N$ number of CR nodes facilitate detection of a potential spectrum hole. Based on the signal that a CR node receives at its input sensing channel, the energy detector comes up with a binary decision about the presence of primary user (PU). These decisions are then conveyed by each node through its output reporting channel to fusion centre (FC). Individual decisions are subjected to fading induced errors which are reduced through data fusion that takes place at FC.

The equivalent baseband received signal at $i$ th energy detector node is

$$
r_{i}(t)= \begin{cases}n_{i}(t) & : \mathcal{H}_{0} \\ h_{i} s(t)+n_{i}(t) & : \mathcal{H}_{1}\end{cases}
$$

where $n_{i}(t)$ is additive white Gaussian noise (AWGN), $h_{i}$ is complex channel fading amplitude, $s(t)$ is transmitted signal with energy $E_{s}$, and $\mathcal{H}_{0}$ and $\mathcal{H}_{1}$ are the null (PU absent) and alternative (PU present) hypotheses, respectively. While the noise, $n_{i} \sim \mathcal{C N}\left(0, \sigma_{n}^{2}\right)$, is zero-mean circularly symmetric complex Gaussian with variance $\sigma_{n}^{2}$, the statistics of fading coefficient, $h=h_{I}+j h_{Q} ; j=\sqrt{-1}, h_{I, Q} \sim \mathcal{N}\left(m_{I, Q}, \sigma_{I, Q}^{2}\right)$ is complex Gaussian but not necessarily symmetric or having zero mean.

Contrary to the conventional energy detectors that works on the principle of simple signal squaring, an IED computes the decision statistic, $W_{i}=$ $\left|r_{i}\right|^{p} ; p>0$. Thus, the conditional probability density function (PDF) of $W_{i}$ under the null hypothesis follows a modified central chi-square distribution [1]

$$
f_{W_{i} \mid \mathcal{H}_{0}}(x)=2 x^{(2 / p)-1} /\left(p \sigma_{n}^{2}\right) \exp \left(-x^{2 / p} / \sigma_{n}^{2}\right)
$$

which is independent of the fading mechanism in the sensing channel. The conditional PDF under hypothesis $\mathcal{H}_{1}$, however, depends on the fading type. In absence of fading, i.e. for an AWGN channel $\left(h_{i}=1\right)$, it follows a modified 
non-central chi-square distribution

$$
\begin{gathered}
f_{W_{i} \mid \mathcal{H}_{1_{G N}}}(x)=2 x^{(2 / p)-1} /\left(p \sigma_{n}^{2}\right) \exp \left[-\left(x^{2 / p}+E_{s}\right) / \sigma_{n}^{2}\right] \\
\times I_{0}\left(2 x^{1 / p} \sqrt{E_{s}} / \sigma_{n}^{2}\right)
\end{gathered}
$$

while for Rayleigh fading channel, the PDF is given by [1]

$$
f_{W_{i} \mid \mathcal{H}_{1}}(x)=2 x^{(2 / p)-1} /\left[p\left(E_{s}+\sigma_{n}^{2}\right)\right] \exp \left[-x^{2 / p} /\left(E_{s}+\sigma_{n}^{2}\right)\right]
$$

Equation (4) may be obtained after expressing the received signal under $\mathcal{H}_{1}$ as a sum of real and imaginary components, i.e. $r_{i}=\left(\sqrt{E_{s}} h_{I}+n_{I}\right)+j\left(\sqrt{E_{s}} h_{Q}+\right.$ $\left.n_{Q}\right)=r_{I}+j r_{Q}$, where $n_{i}=n_{I}+j n_{Q} ; n_{I, Q} \sim \mathcal{N}\left(0, \sigma_{n}^{2} / 2\right)$ and for Rayleigh fading, $\sigma_{I, Q}^{2}=1 / 2$, which implies $r_{I, Q} \sim \mathcal{N}\left(0,\left[E_{s}+\sigma_{n}^{2}\right] / 2\right)$.

In this paper we assume multiple input single output (MISO) CR nodes where a selection combiner (SC) chooses the best input branch out of $M$ available branches with a decision statistics $Z_{i}=\max \left(W_{i 1}, W_{i 2}, \ldots, W_{i M}\right)$. The corresponding conditional cumulative distribution functions (CDFs) under the complementary hypotheses are, $F_{Z_{i} \mid \mathcal{H}_{0}}=F_{W_{i} \mid \mathcal{H}_{0}}^{M}$ and $F_{Z_{i} \mid \mathcal{H}_{1}}=F_{W_{i} \mid \mathcal{H}_{1}}^{M}$ considering independent and identically distributed (IID) fading over all the input antenna branches. From (2), it is obvious that the conditional CDF with $\mathrm{SC}$ under hypothesis $\mathcal{H}_{0}$ is

$$
F_{Z_{i} \mid \mathcal{H}_{0}}(z)=\left[1-\exp \left(-z^{2 / p} / \sigma_{n}^{2}\right)\right]^{M}
$$

On the other hand, the conditional CDFs under $\mathcal{H}_{1}$ in AWGN and Rayleigh faded sensing environments are given by

$$
F_{Z_{i} \mid \mathcal{H}_{1_{G N}}}(z)=\left[1-Q\left(\sqrt{2 E_{s} / \sigma_{n}^{2}}, z^{1 / p} \sqrt{2 / \sigma_{n}^{2}}\right)\right]^{M}
$$

and

$$
F_{Z_{i} \mid \mathcal{H}_{1_{R}}}(z)=\left\{1-\exp \left[-z^{2 / p} /\left(E_{s}+\sigma_{n}^{2}\right)\right]\right\}^{M}
$$

respectively, where $Q(\cdot, \cdot)$ denotes Marcum's $Q$-function. It may be noted here that [3, Eq. (9)] was utilized to obtain (6).

The combiner output in each CR is applied to a one-bit hard decision detector which takes decision about the presence of the PU, i.e. $Z_{i} \underset{0}{\stackrel{1}{\gtrless}} \lambda$, where $\lambda$ is the common detection threshold, $\lambda=\lambda_{n} \sigma_{n}^{p}$ with $\lambda_{n}$ being a fixed normalized detection threshold. Next we invoke the generalized expressions for probabilities of false alarm, $P_{f}=\int_{\lambda}^{\infty} f_{Z \mid \mathcal{H}_{0}}(z) d z=1-F_{Z \mid \mathcal{H}_{0}}(\lambda)$, and missed detection, $P_{m}=\int_{0}^{\lambda} f_{Z \mid \mathcal{H}_{1}}(z) d z=F_{Z \mid \mathcal{H}_{1}}(\lambda)$, from Eq. (41) [4, Chapter 2]. This leads to an independent expression of false alarm probability of each CR

$$
P_{f}=1-\left[1-\exp \left(-\lambda^{2 / p} / \sigma_{n}^{2}\right)\right]^{M}
$$

As the missed detection probability is related to hypothesis $\mathcal{H}_{1}$, we end up with different expressions of $P_{m}$ for sensing channels without (AWGN) and with Rayleigh fading, i.e.

$$
P_{m_{G N}}=\left[1-Q\left(\sqrt{2 E_{s} / \sigma_{n}^{2}}, \lambda^{1 / p} \sqrt{2 / \sigma_{n}^{2}}\right)\right]^{M}
$$




$$
P_{m_{R}}=\left\{1-\exp \left[-\lambda^{2 / p} /\left(\sigma_{n}^{2}(1+\bar{\gamma})\right)\right]\right\}^{M}
$$

where $\bar{\gamma}=E_{s} / \sigma_{n}^{2}$ is the average signal to noise ratio (SNR) of the sensing channel.

We assume the reporting channels between CRs and FC to be identically distributed binary symmetric channels (BSC) with a fixed bit inversion probability of $r$. The overall probability of false alarm $\left(Q_{f}\right)$ and the probability of missed detection $\left(Q_{m}\right)$ under OR-logic fusion at FC is thus, $Q_{f}=1-\left[\left(1-P_{f}\right)(1-r)+r P_{f}\right]^{N}$ and $Q_{m}=\left[P_{m}(1-r)+r\left(1-P_{m}\right)\right]^{N}$, where $P_{m}$ and $P_{f}$ are the pair of missed and false alarm probabilities at each CR, and there are $N$ such CRs present. Finally, the total error rate is given by $Q_{m}+Q_{f}$.

\section{$3 P_{m}$ over Hoyt faded sensing channels}

For Hoyt faded environment, the fading channel coefficients, $h_{i}=h_{I}+j h_{Q}$, are modeled as complex Hoyt processes with the in-phase and quadrature components are given by [5], $h_{I, Q} \sim \mathcal{N}\left(0, \sigma_{I, Q}^{2}\right) ; \sigma_{I}=\sqrt{\Omega q^{2} /\left(1+q^{2}\right)}, \sigma_{Q}=$ $\sqrt{\Omega /\left(1+q^{2}\right)}$, where the average fading power, $\Omega=E\left\{h^{2}\right\}$, is kept normalized to unity, and $q$ is the Hoyt fading parameter that ranges from 0 to 1 .

The conditional PDF of $W_{i}$ under $\mathcal{H}_{1}$ in Hoyt fading is derived using a transformation of random variables and the PDF given in [5] as

$$
\begin{gathered}
f_{W_{i} \mid H_{1}}(x)=y^{2 / p-1} /\left(p \sigma_{1} \sigma_{2}\right) \exp \left[-\left(y^{2 / p} / 4\right)\left(1 / \sigma_{2}^{2}+1 / \sigma_{1}^{2}\right)\right] \\
\times I_{0}\left[\left(y^{2 / p} / 4\right)\left(1 / \sigma_{2}^{2}-1 / \sigma_{1}^{2}\right)\right]
\end{gathered}
$$

where $\sigma_{1}^{2}=E_{s} \sigma_{I}^{2}+\sigma_{n}^{2} / 2$ and $\sigma_{2}^{2}=E_{s} \sigma_{Q}^{2}+\sigma_{n}^{2} / 2$. Using [5, Eq. (58)] and after a few algebraic manipulations, the conditional CDF of the decision statistic at the $\mathrm{SC}$ output is given by

$$
F_{Z \mid H_{1}}(z)=(1 / 16)\left[1+\exp \left(-\mathcal{A} z^{2 / p}\right) I_{0}\left(\mathcal{B} z^{2 / p}\right)-2 Q\left(\mathcal{U}_{1}, \mathcal{V}_{1}\right)\right]^{M}
$$

where, $\{\mathcal{A}, \mathcal{B}\}=(1 / 4)\left(1 / \sigma_{2}^{2} \pm 1 / \sigma_{1}^{2}\right)$ and $\left\{\mathcal{U}_{1}, \mathcal{V}_{1}\right\}=\sqrt{\left(\mathcal{A} \mp \sqrt{\mathcal{A}^{2}-\mathcal{B}^{2}}\right) z^{2 / p}}$.

Finally, replacing the variable in (12) with the threshold $\lambda$ we obtain the probability of missed detection in Hoyt fading environment as

$$
P_{m_{\text {Hoyt }}}=(1 / 16)\left[1+\exp \left(-\mathcal{A} \lambda^{2 / p}\right) I_{0}\left(\mathcal{B} \lambda^{2 / p}\right)-2 Q\left(\mathcal{U}_{2}, \mathcal{V}_{2}\right)\right]^{M}
$$

where, $\left\{\mathcal{U}_{2}, \mathcal{V}_{2}\right\}=\sqrt{\left(\mathcal{A} \mp \sqrt{\mathcal{A}^{2}-\mathcal{B}^{2}}\right) \lambda^{2 / p}}$.

\section{$4 P_{m}$ over Rician faded sensing channels}

For Rician channel, the statistics of the complex fading coefficient components are given by, $h_{I} \sim \mathcal{N}\left(s, \sigma_{h}^{2} / 2\right), h_{Q} \sim \mathcal{N}\left(0, \sigma_{h}^{2} / 2\right)$. The Rician fading parameter $K(\geq 0)$ is the ratio of the direct path signal power to the scattered signal component power, $K=s^{2} / \sigma_{h}^{2}$, while the total fading power is $E\left\{h_{i}^{2}\right\}=s^{2}+\sigma_{h}^{2}$, which contains both the direct and scattered components. Assuming that the fading envelope is normalized, i.e. $E\left\{h^{2}\right\}=1$, we have, $\sigma_{h}=1 / \sqrt{1+K}$ and $s=\sqrt{K /(1+K)}[6]$. 
The statistical characterization of real and imaginary components of the IED input signal is as follows, $r_{I} \sim \mathcal{N}\left(s \sqrt{E_{s}},\left[E_{s} \sigma_{h}^{2}+\sigma_{n}^{2}\right] / 2\right)$ and $r_{Q} \sim$ $\mathcal{N}\left(0,\left[E_{s} \sigma_{h}^{2}+\sigma_{n}^{2}\right] / 2\right)$. This implies that the received signal $r_{i}=r_{I}+j r_{Q}$ surely follows a Rician distribution. The conditional PDF of $W_{i}$, when PU is present, is thus obtained through a transformation of random variable as

$$
\begin{aligned}
& f_{W_{i} \mid H_{1}}(y)=\exp \left[-\left(y^{2 / p}+s^{2} E_{s}\right) /\left(E_{s} \sigma_{h}^{2}+\sigma_{n}^{2}\right)\right] \\
& \times 2 y^{(2 / p)-1} /\left[p\left(E_{s} \sigma_{h}^{2}+\sigma_{n}^{2}\right)\right] I_{0}\left[2 s \sqrt{E_{s}} y^{1 / p} /\left(E_{s} \sigma_{h}^{2}+\sigma_{n}^{2}\right)\right]
\end{aligned}
$$

Next, making use of [3, Eq. (9)], one may get the conditional CDF with SC

$$
F_{Z \mid H_{1}}(z)=\left[1-Q\left(\sqrt{\mathcal{D}_{1} E_{s}}, z^{1 / p} \sqrt{\mathcal{D}_{1}}\right)\right]^{M}
$$

and the probability of missed detection in Rician faded environment

$$
P_{m_{\text {Rice }}}=\left[1-Q\left(\sqrt{\mathcal{D}_{2} E_{s}}, \lambda^{1 / p} \sqrt{\mathcal{D}_{2}}\right)\right]^{M}
$$

where $\mathcal{D}_{1}=2 /\left(E_{s} \sigma_{h}^{2}+\sigma_{n}^{2}\right), \mathcal{D}_{2}=2 /\left[\sigma_{n}^{2}(1+\bar{\gamma})\right]$, and $\bar{\gamma}=E_{s} \sigma_{h}^{2} / \sigma_{n}^{2}$.

\section{Results and discussions}

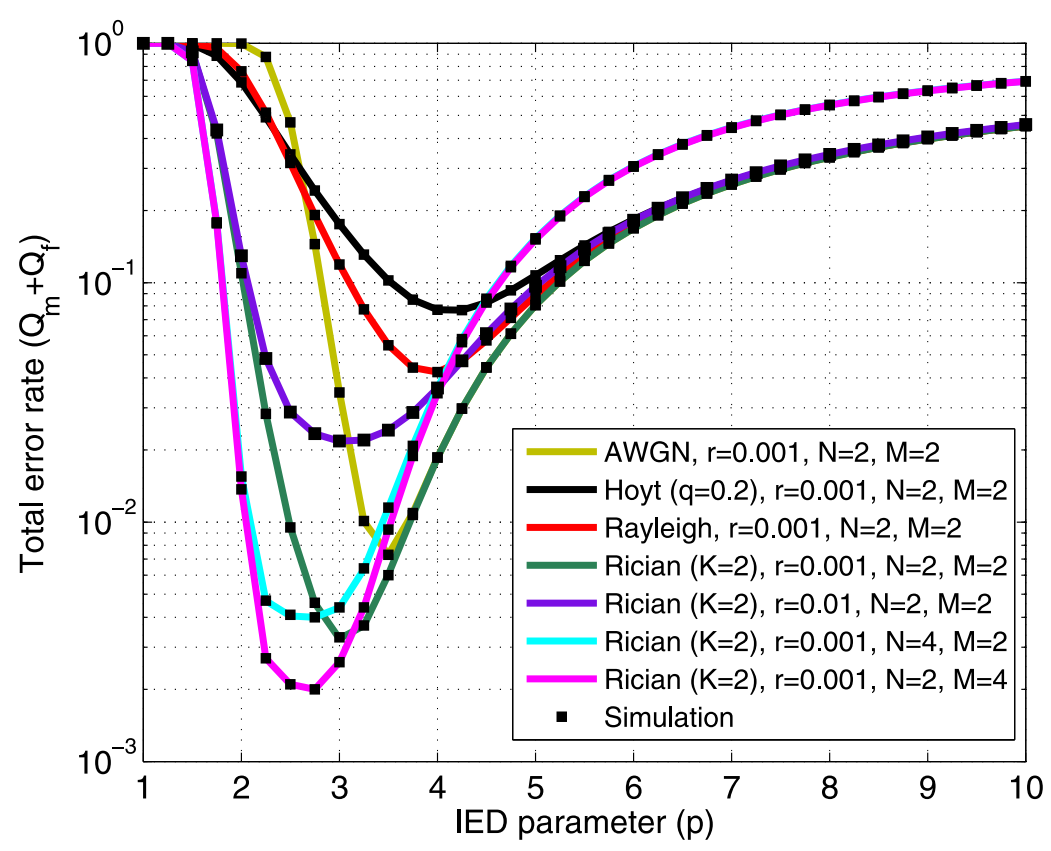

Fig. 1. $Q_{m}+Q_{f}$ versus $p$ for different environments $(\bar{\gamma}=$ $\left.10 \mathrm{~dB}, \lambda_{n}=30\right)$

As seen from Fig. 1, the optimum IED parameter value $\left(p^{*}\right)$ that minimizes total error probability $\left(Q_{m}+Q_{f}\right)$, is more than 2 (conventional detector) for all the different fading channels. The value of $p^{*}$ depends on other CSS parameters, and considering Rician environment as a benchmark, it can be concluded that an increase in either in the number of CRs $(N)$ or in the number of antennas $(M)$ per CR reduces $p^{*}$ (although $p^{*}>2$ ), while the error rate in the reporting channel $r$ does not significantly affect $p^{*}$. Fig. 2 
depicts that there exists an optimum normalized detection threshold $\left(\lambda_{n}^{*}\right)$ as well. Considering Hoyt faded sensing as a test case, one may also observe how $\lambda_{n}^{*}$ varies with $p, N$, or $M$.

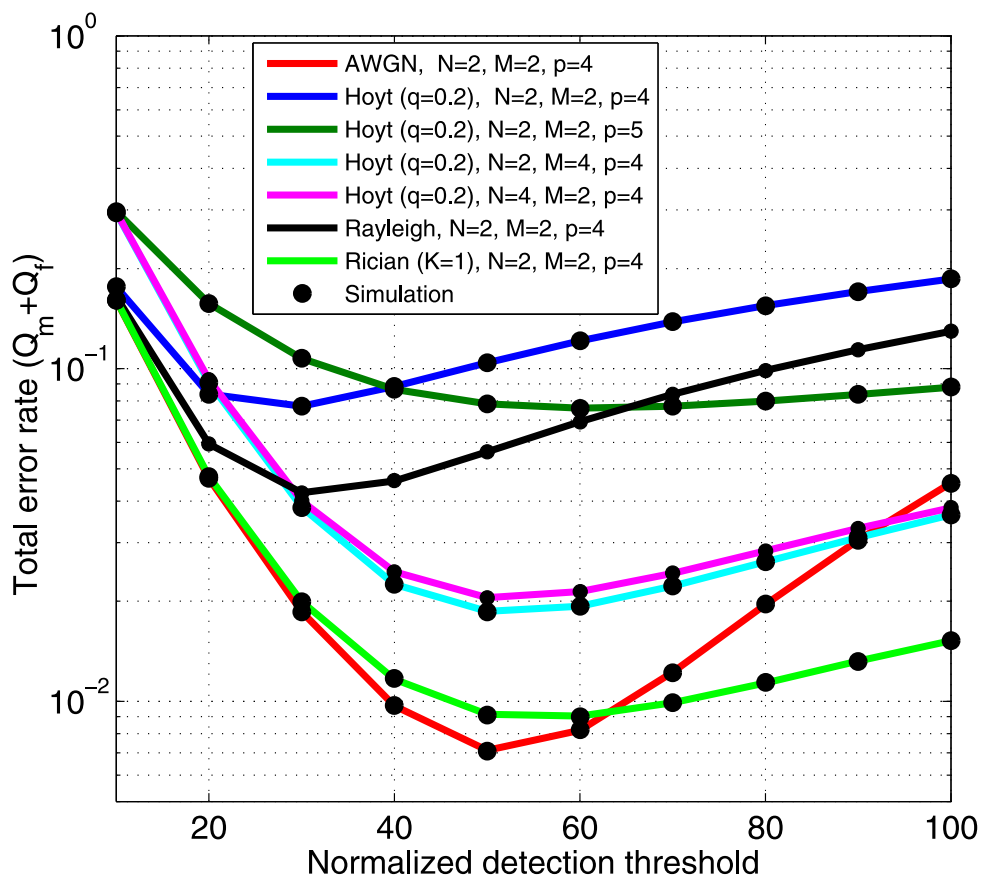

Fig. 2. $Q_{m}+Q_{f}$ versus $\lambda_{n}$ for different environments $(\bar{\gamma}=$ $10 \mathrm{~dB}, r=0.001)$.

\section{Conclusion}

Expressions for probability of false alarm and missed detection have been developed for CSS involving IED based CR with multiple antennas, where sensing channel is Hoyt or Rician faded. Impact of number of cooperating CRs, number of antennas in each CR, and reliability of reporting channel on optimal values of IED parameter and normalized detection threshold have also been assessed. 